\title{
Deployable Structures Mission in Medium Earth Orbit
}

\author{
James Winter, Greg Spanjers, Dan Cohen ${ }^{1}$, Aaron Adler, Shane Kemper ${ }^{1}$, 1Lt Shaun Easley, Keith Denoyer ${ }^{2}$, \\ 2Lt Martin Tolliver, Larry Davis ${ }^{1}$, Jason Guarnieri, and Richard Glover ${ }^{1}$ \\ Space Vehicles Directorate, Air Force Research Laboratory (AFRL), Kirtland AFB, New Mexico \\ james.winter@kirtland.af.mil \\ ${ }^{1}$ Planning Systems Incorporated, Albuquerque, New Mexico \\ ${ }^{2}$ Sequoia Technologies Incorporated, Albuquerque, New Mexico
}

Abstract-The Air Force Research Laboratory (AFRL) Space Vehicles Directorate has developed a mission to research the technologies needed for large space structures, high-power generation, and protection of space assets within the high radiation environment of a medium earth orbit (MEO). The resulting Deployable Structures Experiment (DSX) will address a number of basic science experiments via a single MEO testbed platform. The DSX concept, with specific emphasis on scientific merit, relevance to the Air Force needs, and necessity for flight demonstration, was conceived and proposed for further development in mid-2003. The program then went through 8 months of proposal and review, maturing the overall design, scientific merit and payoff, and Air Force relevance. Ultimately, of the six proposed concepts, DSX was selected in January 2004 as the next AFRL/VS basic research flight experiment. During 2004 the DSX concept was refined, with specific focus on requirement derivation and allocation to hardware and software solutions. The DSX mission, matured in parallel, is the primary subject of this paper.

The baseline DSX experiment should be launch ready by late 2009, with at least one year of on-orbit operations scheduled. DSX comprises five research experiments, that will pave the way for new Department of Defense (DoD) capabilities in space surveillance, microsats with large aperture and power, and protection of space assets from natural and enhanced radiation environments. The DSX experiments include research on:

1. Transformational Deployed Structures: The deployment, dimensional stability, and MEO environment degradation of large deployable space structures

2. Adaptive Control of Structures: The on-orbit system identification (SysID) of the dynamics of these large deployable structures and the use of multiple-inputmultiple-output (MIMO) autonomous adaptive control techniques

3. Radiation Belt Remediation: The physics of Very Low Frequency (VLF, 3 to $30 \mathrm{kHz}$ ) electromagnetic wave injection from ground-based transmitters, propagation, and wave-particle interactions in the magnetosphere relevant to radiation-belt remediation (RBR) techniques

4. Lightweight, High-power, Thin-film Photovoltaics: The performance, radiation tolerance, plasma interactions, and thermal annealing of thin-film photovoltaics (TF-PV) in a $\mathrm{MEO}$ environment

5. Space Weather: The detailed measurement and mapping of energetic particle and plasma distributions in the poorly characterized MEO environment

\section{TABLE OF CONTENTS}

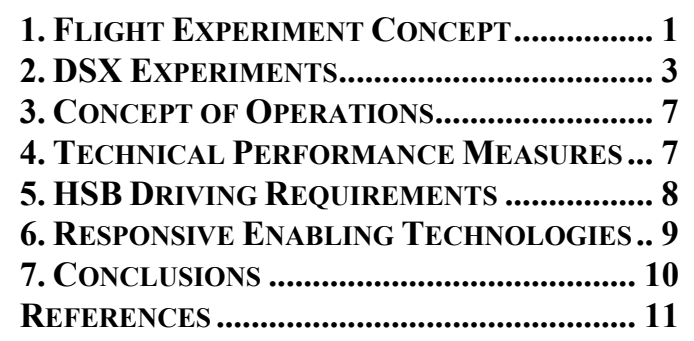

Successful completion of the DSX mission will assist in removing power, aperture, and the MEO environment as significant design constraints for future space systems.

\section{FLIGHT EXPERIMENT CONCEPT}

The functional baseline configuration for the DSX flight experiment is shown in Figure 1. The core is the Evolved Expanded Launch Vehicle (EELV) Secondary Payload Adapter (ESPA) Ring [1,2], used to maximize launch opportunities on both Space Test Program (STP) and operational DoD launchers. Every EELV launch is, therefore, a potential ride for DSX. The ESPA ring, which comprises the primary structure for DSX, is upgraded to provide host spacecraft functions (avionics; telemetry, tracking, and control (TT\&C); attitude determination and control system (ADCS); command and data handling (C\&DH); and power management and distribution) by attaching components to a Host Spacecraft Bus (HSB) and/or the ring interior. The DSX experiment payloads,

"U.S. Government work not protected by U.S. copyright." For U.S. Government employees only. IEEEAC paper \#1531, Paper Version 4, Updated January 21, 2005 
attached to the ring exterior, are designed to be stowed within a 4-m EELV fairing. Unlike the traditional ESPA approach, the DSX experiments and host spacecraft remain attached and do not separate. After the primary satellite is deployed, the DSX ESPA separates from the EELV second stage booster to become a free-flyer spacecraft. The experiment is baselined for flight in the radiation belts, with a $6,000 \times 12,000 \mathrm{~km}$ elliptical orbit, $27^{\circ}$ inclination, and 1 year of mission operations. Figure 2 shows DSX in its stowed-for-launch configuration. DSX has an upper separation interface to the primary payload adapter and a lower separation interface to the EELV upper stage adapter.

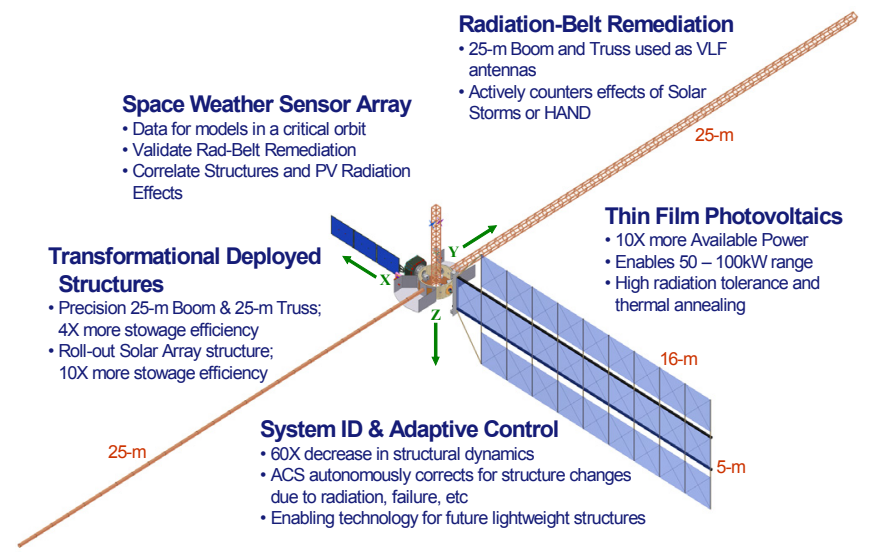

Figure 1 - DSX Flight Experiment Configuration (Deployed).

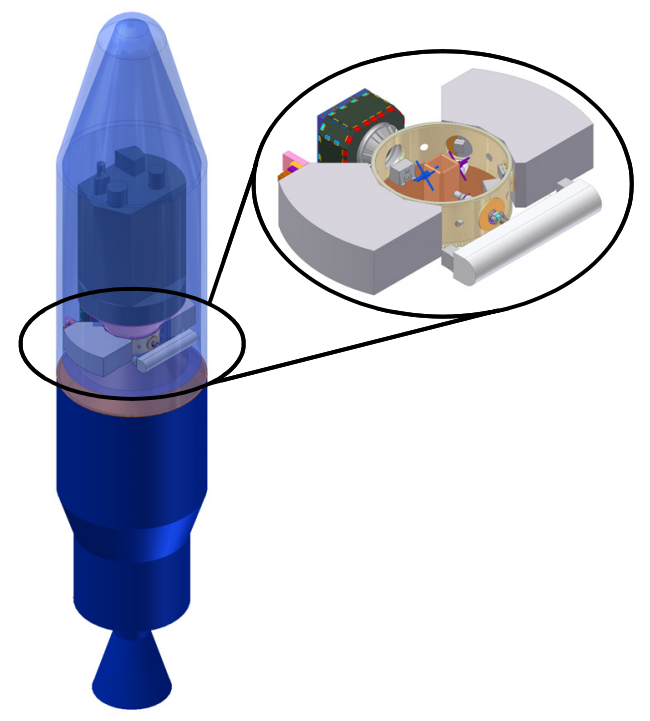

Figure 2 - DSX Stowed for Launch

Figure 3 shows the packaging of DSX equipment with respect to its primary structure, the ESPA ring.

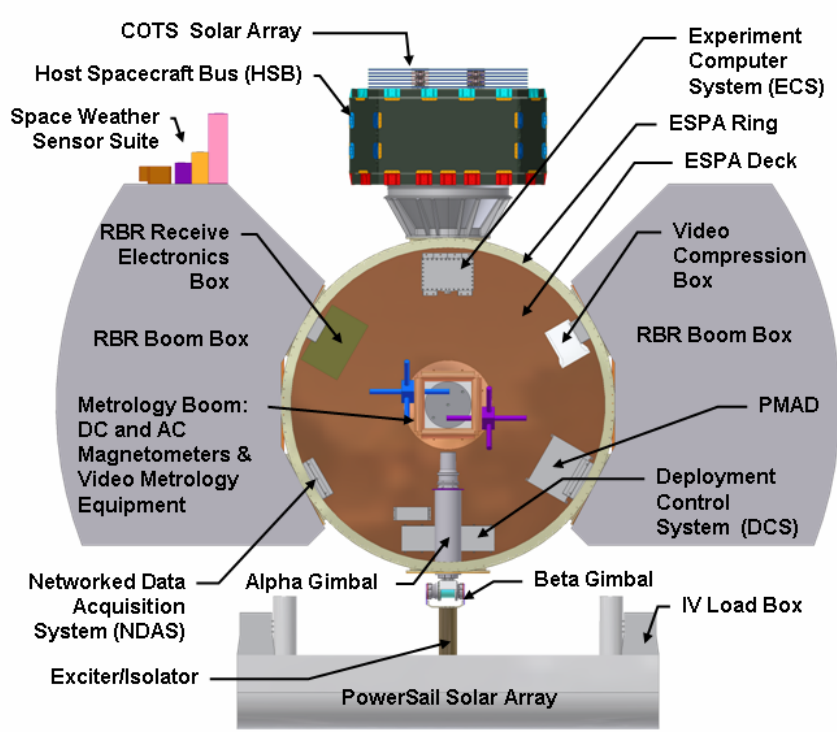

Figure 3 - DSX Equipment Packaging (Stowed)

The DSX ground segment consists of three major elements: mission control, global TT\&C network, and remote experiment sites, as shown in Figure 4. The mission will be operated out of both the Space and Missile Systems Center's Research, Development, Test and Evaluation Support Complex (Det-12/RSC) and AFRL's Missions Operations and Data Center (MOC/DC) facilities located at Kirtland AFB, NM. Det-12/RSC will provide the real-time contacts support, schedule the AFSCN TT\&C network, and acquire both spacecraft and payload data from the space vehicle. Commands will initially be developed from AFRL's MOC/DC facility where the effects will be simulated to validate proper operation. These command modules will be sent to the RSC for uplink via SGLS communication protocol to the DSX satellite. Down-linked information from the space vehicle state-of-health will initially be validated from the real-time operations system located at the RSC.

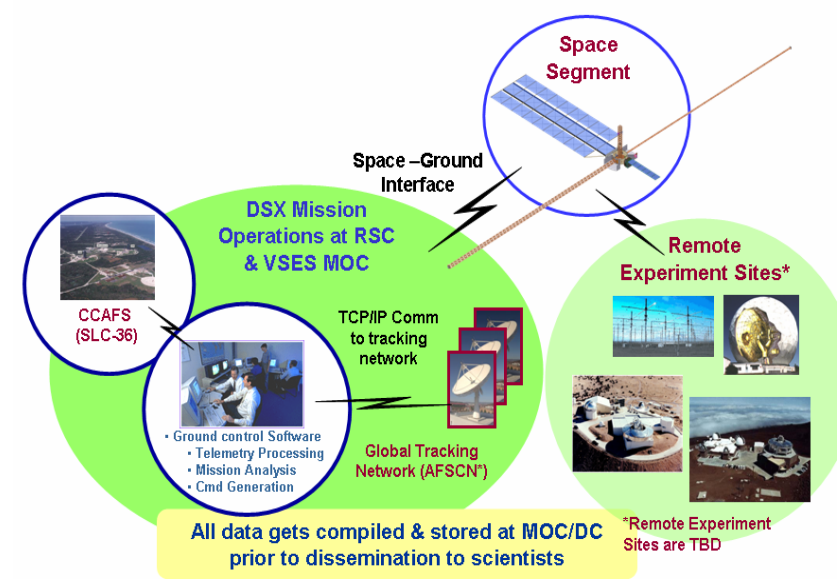

Figure 4 - DSX Ground Segment 
Data from remote experiment sites i.e., optical and radar ranges and VLF ground installations, are coordinated at the data center. All DSX data products, including real-time telemetry, stored state-of-health, stored and real-time experiment data, and ranging information, will be sent to the MOC/DC for further analysis and data archival. Experiment data and required spacecraft byproducts will be distributed to each experiment team.

\section{DSX EXPERIMENTS}

\section{A. Lightweight Deployable Structures, System ID, and MIMO Adaptive Control}

Current space structures are often developed with a buildtest-build-test strategy. Large design margins are used to offset the technical risk inherent in the lack of scientific understanding of the critical physical processes. DSX, using a balanced approach of ground testing, finite-element (FE) model development, and flight experiments to understand the fundamental processes, will therby enable low-mass, high-reliability deployed space structures for future DoD assets.

Deployable space structures use a variety of deployment techniques: mechanical systems deployed with motors, self-deploying structures using stored strain energy, other mechanical approaches, inflatable, and inflatable-rigidizable systems. Despite the range of technology options, DSX will address questions fundamental to all space structures.

For example, joints remain a significant issue. Large deployable structures must be bent, folded, or otherwise contorted to fit into the fairing for launch. Mechanical joints can be used between composite or inflatable struts. Torque imbalances, joint friction, and joint nonlinearity will all contribute, however, to unpredictable deployment behavior. [3] Due to their lack of moving parts, shapememory composite materials are attractive joints; however, they suffer from fiber breakage during stowage, torque imbalances, and unknown frictional effects. More mature shape-memory alloy materials lack the high stiffness needed for precision applications. Fold effects, such as damping, friction, local yielding, and stored strain energy, typically change when a joint is connected to a strut. Due to the inability to realistically gravity off-load the strut, errors introduced in ground-based tests are larger that these effects on orbit. Some inflatables eliminate moving joints by folding the inflatable strut itself. Unfortunately, such folding results in fiber breakage and deformation that weakens the material in a currently unpredictable fashion.

The primary hypothesis for the DSX structures experiments is that the point-specific gravity off-loading of ground testing, internal material stresses caused by packaging, and nonlinear joint effects cause sufficient uncertainty in the finite-element (FE) model to preclude the use of large deployable structures in future DoD space assets. The deployable structures approach on DSX is to perform the basic science needed to enable a predictive design capability to achieve mass and volume optimization for future deployable space structures.

The objectives for deployable structures are to determine if the deployment kinematics of large space structures, the onorbit behavior (structural dynamics, precision) of deployed structures, and the effects of the space environment on large deployed structures can be predicted and modeled. The planned flight experiments consist of 0 -g deployments of structures (video, accelerometers, photogrammetry), dynamics of deployed structures (system ID and accelerometer), and dynamic changes over time in MEO environment (system ID, position, and temperature). Extensive ground experiments and Modeling and Simulation activities will be used for comparison to the flight data, and complete the approach to meet the deployable structure objectives.

The DSX structures experiments consist of (1) two 25-m class booms that will also serve as the low-impedance VLF antenna for RBR VLF and (2) a low-precision surface structure known as PowerSail, also used to support the TFPV solar cell experiments.

The specific design and manufacture of the boom structures will be determined through a competitive source-selection in FY04-FY05. The AFRL intent is to do basic research on structures attractive for future large-aperture surveillance applications, such as a MEO-based Space Based Radar (SBR). To this end, DSX will seek to perform research on the structure concepts in development under the DARPA Innovative SBR Antenna Technologies (ISAT) program in an effort to achieve significant risk reduction for a MEO SBR system.

Whereas the deployed booms are designed for precision applications requiring high rigidity, the PowerSail array [4] uses a depthless, large-area structure that greatly reduces system mass at the expense of rigidity. The surface structure is attractive for solar array applications where mass is a primary system design driver, and the increased dynamics associated with the structure have minimal impact on the power generation capability.

A key challenge of the PowerSail roll-out array is deployment of large-area 2-D structures based on flexible membranes. Deployment is accomplished using shape memory longerons and transverse composite tensioning members bonded to the backside of the Kapton flex circuit. Sequential heating of shape memory actuators allows the 
array to unroll at a controlled rate and lock into a relatively high stiffness structure (Figure 5a). Bilateral wings unfold from the central member via edge mounted shape memory hinges (Figure 5b). Each deployment system relies on recovery of the shape memory actuator to a high-stiffness geometry following removal of the heater power. Thermistors placed between thin film heaters and the shape memory foils provide a temperature feedback signal to a deployment controller. Upon reaching the glass transition temperature the controller reduces current to prevent overheating the hinge. Incrementally increasing temperature set points regulates the deployment rate and deployment sequence.

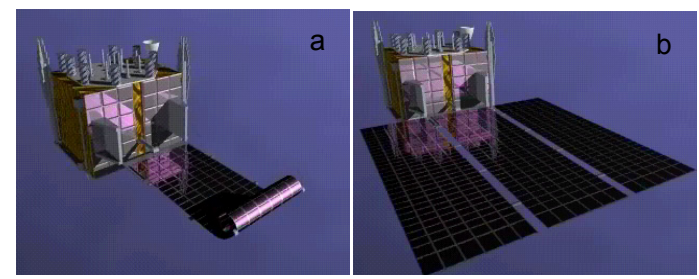

Figure 5 - Rollout Solar Array on DSX

\section{B. VLF Propagation}

The inner magnetosphere $(<10,000 \mathrm{~km})$ is the region where the radiation belts produced by a high-altitude nuclear detonation (HAND) are the most long-lived. Energetic electron belts produced by these events would dominate the radiation environment and significantly degrade Low Earth Orbit (LEO) assets. Methods are being investigated to reduce the intensity of a HAND belt by transmitting VLF electromagnetic waves that will pitch-angle scatter the electrons via cyclotron-resonant wave-particle interactions. Properly directed, this electron scattering will increase the electron velocity parallel to the local magnetic field and lower the altitude of their magnetic reflection. If the magnetic reflection is lowered to altitudes within the upper atmosphere, collisions with the plentiful neutral particles will lead to a depopulation (or "remediation") of the radiation belt along that magnetic flux tube. These processes can be viewed as accelerating the natural rate of electron loss by deliberately enhancing the VLF energy density.

Natural sources of VLF in the magnetosphere include magnetospheric hiss generated by space weather processes and lightning-induced whistler waves propagating along field-aligned ducts through the magnetosphere. To match current models of radiation belt dynamics to the observed behavior of electrons produced by the STARFISH exoatmospheric nuclear blast in 1962, it is necessary to postulate that man-made VLF leaking into the magnetosphere from ground-based submarine communications systems is also a significant source. Direct measurements of VLF power have been sparse because scientific satellites with the required capability (e.g., AF CRRES [5] and NASA IMAGE [6] missions) have been in highly elliptic orbits with apogee $>6$ Earth radii and consequently spend most of their time outside the inner belt and slot region. Placing a VLF transmitter and receiver on the DSX satellite will allow quantitative determination of VLF injection efficiency from a space-based platform and the current levels of natural and man-made VLF waves in the inner magnetosphere. Models of ground-based VLF injection and global magnetospheric propagation will also be validated in a controlled manner. An electron loss cone detector on DSX will provide the capability to directly correlate changes in energetic particle distributions with injected wave power. Accurate models of the VLF injection, propagation, and wave particle interaction processes are critical to assess the viability of an operational RBR system. Measurements of the VLF wave field will also accelerate the development of the natural radiation belt forecast models needed for space situational awareness and mission planning.

The baseline VLF payload on DSX will be a passive receiver to measure the natural and man-made ELF, VLF, and LF signals in the inner magnetosphere in the range of $10 \mathrm{~Hz}$ to $100 \mathrm{kHz}$. AFRL is working to add a transmitter to the DSX baseline and broadcast up to the kilowatt level in the range 1 to $50 \mathrm{kHz}$ and at much smaller levels from 50 to $750 \mathrm{kHz}$. Current designs indicate the instrument would be composed of four components: (1) tri-axial search coil magnetometers located at least $2 \mathrm{~m}$ from the spacecraft with frequency response $10 \mathrm{~Hz}$ to $20 \mathrm{kHz},(2)$ an electric dipole antenna systems consisting of one 50 -m tip-to-tip electric dipole antenna with transmit and receive capability (1 to $800 \mathrm{kHz}$ ), and (3) receiver electronics. AFRL is considering adding transmitter capability, including dynamic tuning circuits. Deployable booms will support the DSX VLF antenna, as shown in Figure 1. The electron loss-cone particle detector will be optimized to view in a relatively narrow solid along the magnetic field lines with a large geometric factor in the $100 \mathrm{keV}$ to $1 \mathrm{MeV}$ range in order to measure electrons scattered out of the belt by VLF processes.

\section{Thin-film Photovoltaics}

No solar array built on thin-film photovoltaic (TF-PV) technology has flown in space. Low power $(<1 \mathrm{~kW})$ TF-PV arrays were developed for TechSat 21 but have not yet flown. [7,8] TF-PV coupons were flown on NASA EO-1, PASP-Plus (APEX Spacecraft 1994), and Mir Space Station, but they were small-scale experiments that did not provide sufficient risk reduction for the TF-PVs to achieve technology transition. 
TF-PV cells offer great promise for power generation on future spacecraft. Although the efficiency of the cells is currently low compared to traditional crystalline cells, these cells are attractive at the system level due to significant increases in power density $(\mathrm{W} / \mathrm{kg})$ and packaging $\left(\mathrm{W} / \mathrm{m}^{3}\right)$. For high-power applications, the greatest TF-PV impact will be the reduced cost. Crystalline cell sizes are limited by the size of the Chemical-Vapor Deposition (CVD) chambers to about $8 \times 8 \mathrm{~cm}$. Assembling the relatively small cells into large blankets requires a large degree of hand labor, driving up the cost. The current cost for high-quality crystalline cell arrays is about $\$ 1,000 / \mathrm{W}$. Under AFRL programs, United Solar has fabricated amorphous silicon (a:Si) TF-PV cells as large as $24 \times 36 \mathrm{~cm}$, which greatly reduces the hand-labor associated with integrating the cells into a blanket. Large quantities of TF-PVs can also be made via a roll-to-roll process, further driving down the cost. The estimated cost for a solar array based on TF-PVs is $\$ 250 / \mathrm{W}$. For a $30-\mathrm{kW}$ array, array cost decreases from $\$ 30 \mathrm{M}$ to $\$ 7.5 \mathrm{M}$.

For applications such as GPS, second-generation SBR in MEO, operational RBR, and LEO-GEO orbit transfers, the greatest benefit of TF-PVs may be their exhibited high radiation tolerance and their ability to anneal the damage due to radiation. Ground tests have shown that TF-PVs degraded by radiation exposure will recover a significant fraction (up to $99 \%$ ) of their performance when subjected to elevated temperatures $\left(\sim 60\right.$ to $\left.80^{\circ} \mathrm{C}\right)$. These tests, however, are fraught with uncertainty due to the many factors involved and possible synergistic influences between effects, such as temperature, radiation, materials, and photon flux.

The hypothesis of the DSX TF-PV experiment is that multiple influences couple in a non-linear fashion to affect the TF-PV performance. The goal of the experiment is to couple ground and flight tests to develop a model for TFPV performance usable in the design of future DoD satellites. The research goal is to determine how the multiple factors affecting TF-PVs combine to change their performance. Space environmental effects on TF-PV systems must be well understood before the potential benefits to operational space power systems can be realized. Ground testing of TF-PVs subjected to various space environmental conditions is currently planned or under way. Testing includes space radiation, UV exposure, micrometeorite impacts, and space plasma interactions. The majority of this ground testing will be performed "sequentially" with only one space environmental condition present at a time.

While this sequential testing will go a long way toward predicting device performance on orbit, its predictive capability assumes that the effect of the various space environmental phenomena on device performance is not synergistic (interdependent). For example, ground testing can be performed for TF-PV devices subjected to single energy level particles at accelerated dosage rates in a sequential fashion, but experimental facilities that can perform concurrent testing with the full spectrum of particle energies at near the actual dosage rate, while the sample is being illuminated with an AM0 solar spectrum and held at operational temperature, are non-existent and believed to be cost prohibitive to construct. A space experiment with TFPV devices, coupled with on-going ground tests, will provide the data needed to determine the interdependency of the space environment influences.

The potential synergistic effect of space environmental factors is particularly critical to address for TF-PV in view of their relatively low-temperature, radiation-damage annealing properties (conventional crystalline PVs do exhibit some annealing but at much higher temperatures not typical for operational spacecraft). Both a:Si and Copper Indium Gallium Diselenide (CIGS) TF-PV devices have demonstrated the ability to self-repair (anneal) at a substantial rate when subjected to operating temperatures of 60 to $80^{\circ} \mathrm{C}$. Ground experiments have also shown that light illumination of CIGS devices may accelerate annealing, but the relative contributions of temperature and light to the process is not clear.

DSX proposes to test multiple configurations of thin-film PVs using diagnostic electronics controllers and plasma modelers similar to those used in the PASP-Plus experiment. [9] In addition, the DSX experimental package will include radiation sensors and controlled heaters to test the predicted temperature dependence of TF-PV annealing rates for radiation-induced damage. For the DSX flight, the PowerSail structure will be populated with TF-PV technologies from multiple vendors and interconnected for both low- and high-voltage operation.

The second TF-PV objective is to determine the effect of the natural radiation environment on thin-film PV coatings. Thin, flexible coatings deposited on thin-film solar modules are intended to replace the thick cover glasses currently used to protect crystalline solar cells. The coatings are designed to protect the TF-PV cells from low-energy proton damage and high-voltage arcing, in addition to providing thermal control. The coatings must be highly transparent to maximize TF-PV performance. The coatings themselves may, however, be radiation damaged. Ground testing can approximate the space radiation and thermal environment through sequential electron, proton, and thermal exposures; however, synergistic effects of simultaneous bombardment of the entire energy spectrum of charged particles, combined with thermal cycling, may result in effects not observed during ground testing. To isolate the effects of 
coating darkening from radiation effects on the thin-film solar cell, the coatings will be applied to triple junction (TJ) solar cells of known radiation response. To determine the loss of transparency in the coatings, the power degradation of the coated TJ solar cells on-orbit will be compared to the predicted $\mathrm{TJ}$ solar cell response.

\section{Space Weather Experiments}

With an orbit between 6,000 and $12,000 \mathrm{~km}$, DSX will explore a large swath of the inner magnetosphere, in particular the outer region of the inner proton belt known as the "slot region," and inner regions of the outer electron belt. This domain has remained largely unexplored due to the understandable tendency of military, commercial, and scientific satellite systems to be located outside the intense regions of radiation, most often at LEO or GEO. Increasing demands for uninterrupted coverage of the Earth from space are, however, driving planners to consider putting satellite constellations in orbits that spend significant time in the inner magnetosphere. Current standard space particle models (e.g., AE-8 and AP-8) can be off by as much as $50 \mathrm{X}$ or more in estimating the time taken to reach hazardous dose levels in the MEO regime. It is imperative that measurements be made of the plasma and energetic particles so that adequate climatological, situational awareness, and forecast models can be developed to enable the successful design and operations of systems in these new and desirable orbit regimes. Accurate environment determination is especially critical on DSX in order to quantify and understand the performance of the structures and TF-PV experiments over the course of the mission.

Deficiencies of current standard radiation belt models in the inner magnetosphere include the lack of (1) spectrally resolved, uncontaminated measurements of high-energy protons $(10$ to $400 \mathrm{MeV})$ and electrons (1 to $30 \mathrm{MeV}$ ) and (2) accurate mid- to low-energy $(<1000 \mathrm{keV})$ measurements of the energetic particle and plasma environment. The space weather instrumentation payload on DSX will address these deficiencies. Electron and proton detectors will measure both the spectral content and angle of arrival of both species over broad energy ranges. An on-board magnetometer will allow for the transformation of angle-of-arrival measurements into estimates of the flux distribution with respect to the local pitch-angle (i.e., the angle between the particle velocity and magnetic field). Local pitch-angle distributions can then be used to estimate global particle distributions by mapping techniques using the well-known equations of motion and magnetic field models tagged to the local measurements. The nominal space weather sensor suite capabilities are described in detail below.

High-energy Proton Spectrometer (HEPS) - HEPS will measure the high-energy proton population in the radiation belts and during solar particle events. The instrument will measure proton energy in the range of 20 to $440 \mathrm{MeV}$ in approximately 20 logarithmically spaced channels. This particle population is not isotropic and must be measured in sufficient angular resolution to image the loss cone, which is on the order of 10 degrees. To do this, the sensor will reconstruct the pitch angle distribution with an accuracy of 7 to 12 degrees (opening half angle). The DSX spacecraft is not a spinner, and the pitch angle measurements must be made using the sensor's field-of-view alone. The sensor must be able to make accurate measurements (less than $10 \%$ contamination) in presence of high-energy electron population in the outer belt and during solar particle events.

High-energy Electron Telescope (HEET)—HEET will measure, without contamination, the high-energy electron population in the radiation belts and during solar particle events in the presence of a large high-energy proton background. The energy range of the measurement will be in the 3 to $30 \mathrm{MeV}$ range, with an expected 10 degree fieldof-view providing pitch-angle resolution.

Low-energy Imaging Particle Spectrometer (LIPS) - LIPS is designed to measure the ring current particles that are important in the energy flow processes in the magnetosphere. This particle population plays an important role in spacecraft charging and surface damage due to sputtering. The instrument, built by Physical Sciences Inc., uses specially designed combinations of scintillator materials to detect electrons and protons with energies between $20 \mathrm{keV}$ and $1 \mathrm{MeV}$. Eight $10 \times 8$ degree field-ofview windows will provide pitch angle resolution.

Compact Environmental Anomaly Sensor (CEASE III) Composed of two dosimeters, two particle telescopes, a single-event effect detector, and two distributed electrostatic analyzers, CEASE III can monitor a broad range of space hazards from surface damage $(100 \mathrm{eV}$ protons and electrons) and charging ( $\mathrm{keV}$ electrons) to single-event effects resulting from $>100 \mathrm{MeV}$ cosmic rays and solar protons. CEASE III extends the capabilities of the original CEASE detector $[10,11]$ by adding two distributed electrostatic sensors that extend the low-energy detection range from $50 \mathrm{keV}$ to approximately $10 \mathrm{eV}$ for both electrons and ions. This activity allows for detailed characterization of the surface-charging and thin-film degrading environments. Spacecraft frame potentials can also be determined from $100 \mathrm{eV}$ to several $\mathrm{keV}$. The angular field-of-view for CEASE is relatively large and will not allow for pitch angle resolved measurements.

DC Magnetometer-A DC fluxgate magnetometer will be used to determine the direction of the magnetic field to better than one degree at all points in the orbit. This value 
will allow the mapping of locally measured particle distributions to global distributions.

\section{CONCEPT OF OPERATIONS}

The DSX mission starts with launch as a secondary payload on an EELV. Following separation of the primary payload from atop DSX, the option may exist, depending on the need to obtain different orbital parameters to re-start the EELV upper stage for repositioning. Following injection into the desired orbit, DSX then separates from the upper stage. Upon detection of the separation event, DSX will undergo a series of autonomous scripted commands to initialize the bus. After acquisition of signal from the ground and verification of nominal operation, the primary host spacecraft bus array deploys and the attitude control system is activated to orient the spacecraft into a powerpositive attitude. Ground controllers will then verify the spacecraft state of health before initiating the instrument deployments. When ready, one or more booms is deployed for the deck panel of the ESPA ring. These booms support a video imaging system used to observe deployment of the experimental structures, science magnetometers, and an optical metrology system. The video imaging system is activated prior to deployment of the experimental structures, the two 25-m RBR booms, and the PowerSail array, as illustrated in Figure 6.

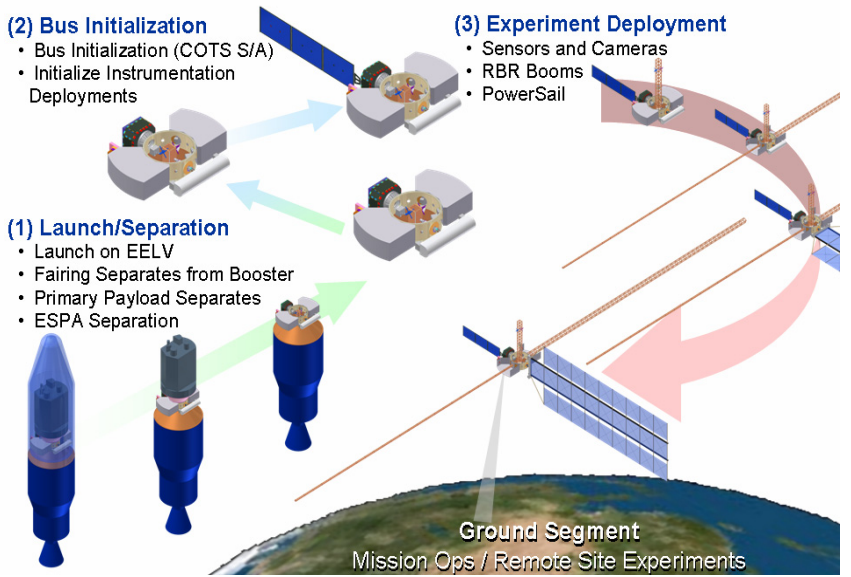

Figure 6 - CONOPS of Early Operations

During deployments the attitude control system is placed in a less responsive mode in consideration of the fact that, during deployments, the center of pressure and moments of inertia will vary significantly. Upon completion of these deployments, additional in-orbit tests are performed before the spacecraft assumes an operational mode as summarized in Figure 7. Scheduling of the sequence of events from separation through entering the operational phase is being developed; it is expected to be accomplished in less than one week.

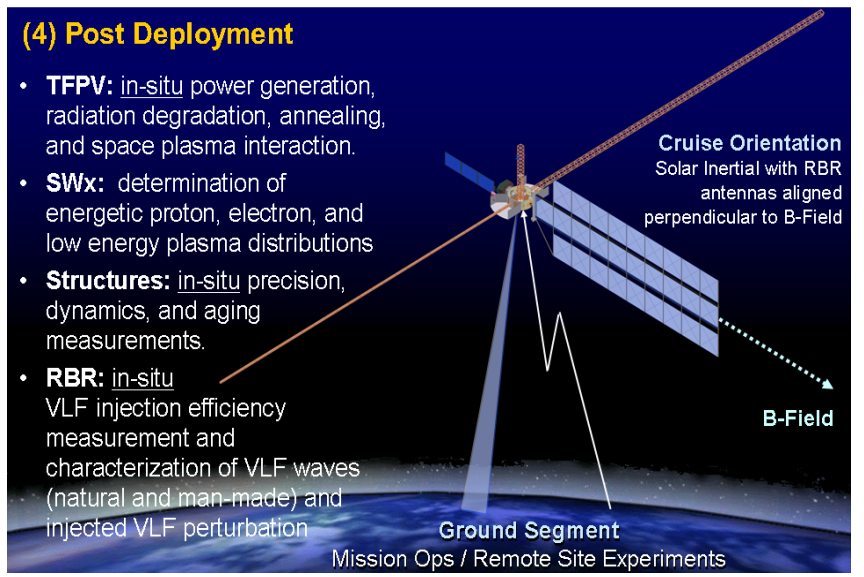

Figure 7 - CONOPS of Operational Phase

\section{Technical Performance Measures}

Since its earliest stages of development, the DSX program has implemented a mass and power control process, and carefully tracked these attributes. The upper mass limit requirement for DSX, in the absence of a manifested launch vehicle with identified throw weight margins, was simply determined as the mass of a fully loaded ESPA ring, or $1205.63 \mathrm{~kg}$. A master equipment list was then developed identifying every equipment or structural element of the spacecraft, with mass and power consumption estimates identified. These budgets are presented in Tables 1 and 2, respectively. The current DSX mass estimate of $733 \mathrm{~kg}$ allows $473 \mathrm{~kg}$ of unallocated mass margin to the above described ESPA limit. 


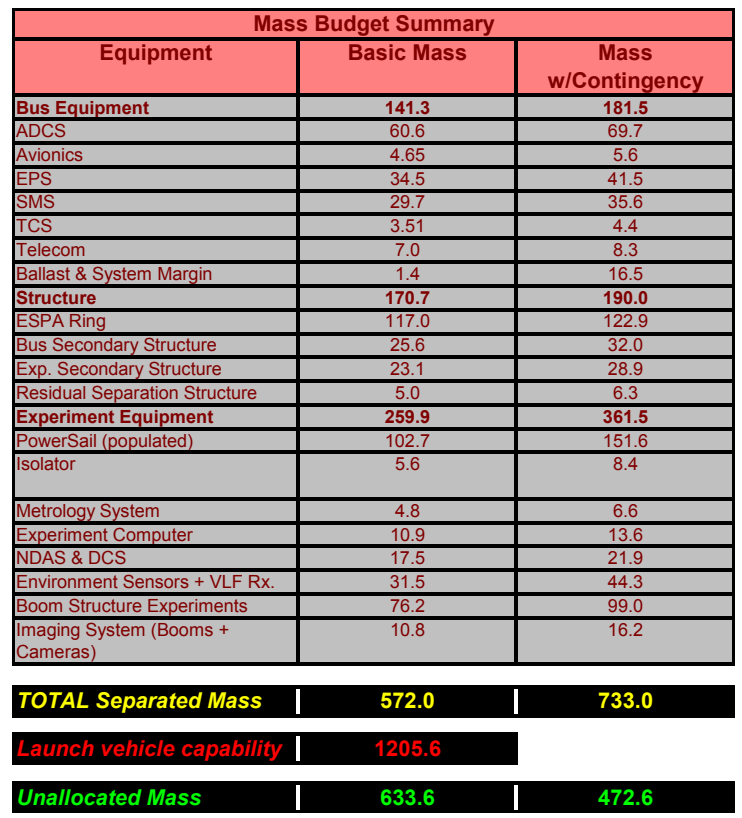

Table 1 - DSX Mass Budget Summary

\begin{tabular}{|c|c|c|}
\hline \multicolumn{2}{|c|}{ Power Budget Summary } & \\
\hline Equipment & $\begin{array}{l}\text { Power } \\
\text { (W) }\end{array}$ & $\begin{array}{c}\text { Power } \\
\text { w/Contingency }\end{array}$ \\
\hline Bus Equipment & 154.2 & 206.3 \\
\hline ADCS & 59.0 & 74.1 \\
\hline Avionics & 72.0 & 90.0 \\
\hline EPS & 0.0 & 0.0 \\
\hline SMS & 0.0 & 0.0 \\
\hline TCS & 8.0 & 22.5 \\
\hline Telecom & 15.2 & 19.7 \\
\hline Experiment Equipment & 250.9 & 322.3 \\
\hline Isolator & 20.0 & 28.0 \\
\hline Metrology & 16.0 & 24.0 \\
\hline ECS & 52.0 & 62.4 \\
\hline NDAS \& DCS & 68.0 & 85.0 \\
\hline Environment Sensors \& VLF & 31.9 & 47.9 \\
\hline PowerSail & 63.0 & 75.0 \\
\hline Boom Experiments & 0.0 & 0.0 \\
\hline Impulsive Power Requirements & 349.7 & \\
\hline PowerSail Deployment (6 stages) & 60.0 & \\
\hline Boom Exp. Deployments & 200.0 & \\
\hline DCS (during deployments) & 10.0 & \\
\hline COTS Boom Deployments & 22.5 & \\
\hline Imaging System & 57.2 & \\
\hline
\end{tabular}

Steady State Total Power

405.1

\begin{tabular}{l|l} 
Maximum Peak Power & $\mathbf{6 7 5 . 4}$
\end{tabular}

Table 2 - Power Budget Summary

\section{HSB DRIVING REQUIREMENTS}

The DSX science objectives impose certain mission-specific driving requirements on the Host Spacecraft Bus (HSB) design. These are identified below.
The nominal orbit $(6000 \times 12000 \mathrm{~km}$ at 27 degree inclination), implies a fairly severe radiation environment, resulting from the orbit residing in the upper Van Allen belts. The Total Ionizing Dose (TID) is equivalent to 690 krad unshielded, or $10 \mathrm{krad}$ with a radiation dose margin (RDM) of 2 with $12 \mathrm{~mm}$ of aluminum absorber shielding. The results of our radiation dose analysis, performed for a worst-case inclination of 0 degree is shown in Figure 8. The implication of these results is that all DSX equipment must be designed to survive the radiation environment through the use of shielding, selection of radiation and SEU tolerant or hardened components, or a combination of these approaches.

With respect to flight orientation, the attitude control system must provide two- or three-axis stabilization, maintain a power positive attitude throughout the mission, and orient the spacecraft such that the RBR/VLF antennas are aligned in a sense normal to the local magnetic field lines for the time in which the spacecraft is within \pm 20 degrees of equatorial (i.e., when VLF propagation experiments and measurements will be performed). Pointing requirements are fairly relaxed, with a 2 degree control and a 1 degree knowledge requirement deemed sufficient.

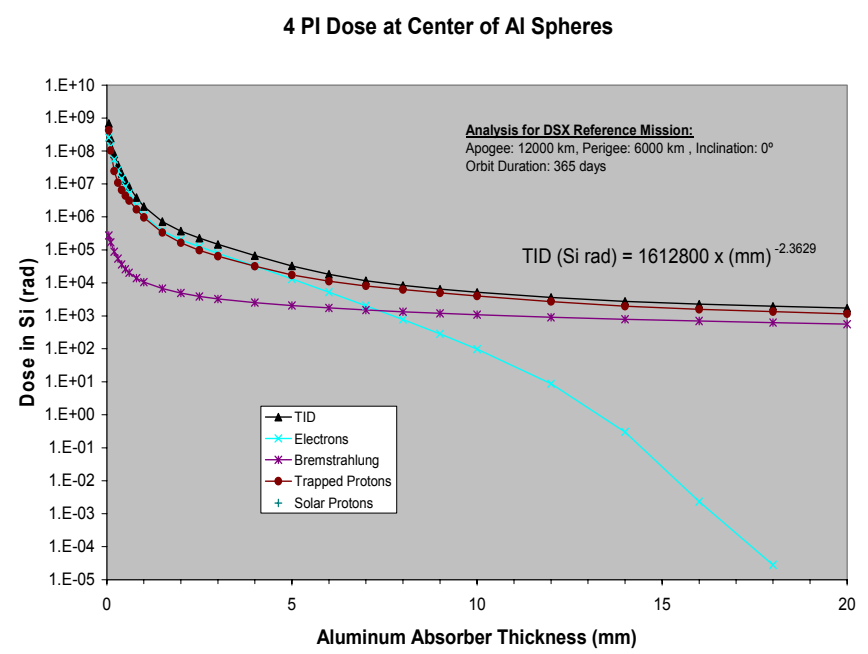

Figure 8 - Total Ionizing Dose Analysis for DSX

Because of resource limitations, the individual experiments on DSX do not run continuously. Moreover, some of the experiments have environmental constraints that limit when useful measurements can be made. As indicated in the preceding discussion, the RBR/VLF measurements may be made only within a limited latitude range. The PowerSail photovoltaics measurements may be made only when the PowerSail array is illuminated. The structures system identification and adaptive controls experiments have no constraints and may be performed at any time when 
spacecraft resources are available (i.e., when RBR and photovoltaics measurements are not being made). The space weather measurements also have no external constraints, and in fact we plan to conduct continuous space weather measurements over the course of the mission. Finally, the critical deployments for which video imagery is required may be performed only when lighting conditions are favorable.

\section{RESPONSIVE ENABLING TECHNOLOGIES}

Historically, spacecraft payloads have been produced via end-to-end development of the entire payload system, as well as significant development of key satellite bus components with interfaces to the payload such as the flight computer and power management system. As part of this effort, a considerable fraction of the engineering development required to produce a working system is devoted to specifying, developing, and testing the components designed to perform payload functions that, in general, fall under the category of payload interface electronics for data acquisition and control. This approach is not conducive to rapid response missions because it depends on monolithic integration methods. Monolithic integration of a satellite involves simultaneous assembly of many components. Such integration can present an "integration and test nightmare," where debugging problems found in system level testing and routine maintenance of the vehicle can be very complex to perform.

Modular integration, on the other hand, allows major subsystems and payload modules to be fully integrated and functionally tested separately. System level testing can be performed in a "FlatSat" configuration, pre-integration where the subsystems are wired together using test harnesses to verify connectivity, as well as performing closed-loop performance tests. This procedure allows for much faster isolation of problems and shorter integration spans.

DSX maximizes the benefits of modular integration via an architecture that consists of:

- A common redundant network infrastructure, or Network Data Acquisition System (NDAS), integrates spacecraft subsystems, components, sensors, actuators, and payload equipment to the avionics core (spacecraft flight computer).

- All devices adhere to a standard interface for control and communications (electrical, power, command and communications protocol).

- Remote network nodes are distributed for point-of service connectivity with equipment (spacecraft subsystems or payloads).
- A dual-redundant network interface card (NIC) in the flight control computer chassis provides a command and data interface between the flight control computer and all items connected to the network.

These elements are shown in Figure 9. Rather then a single on-board computer responsible for handling all spacecraft functions, DSX unburdens the main spacecraft computer with the payload-related operations by providing a dedicated payload interface computer, or Experiment Computer System (ECS) (Figure 10). ECS was chosen because it decouples the requirements of the flight computer from the payload design, allowing for a completely standardized spacecraft flight computer, almost independent of payload requirements. The highly capable ECS design is also generalized in nature so that it can handle a wide range of payload types and classes.

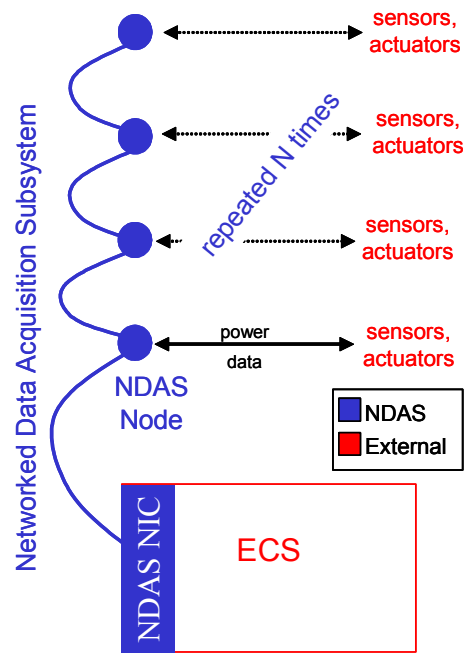

Figure 9 - DSX Data Acquisition Architecture

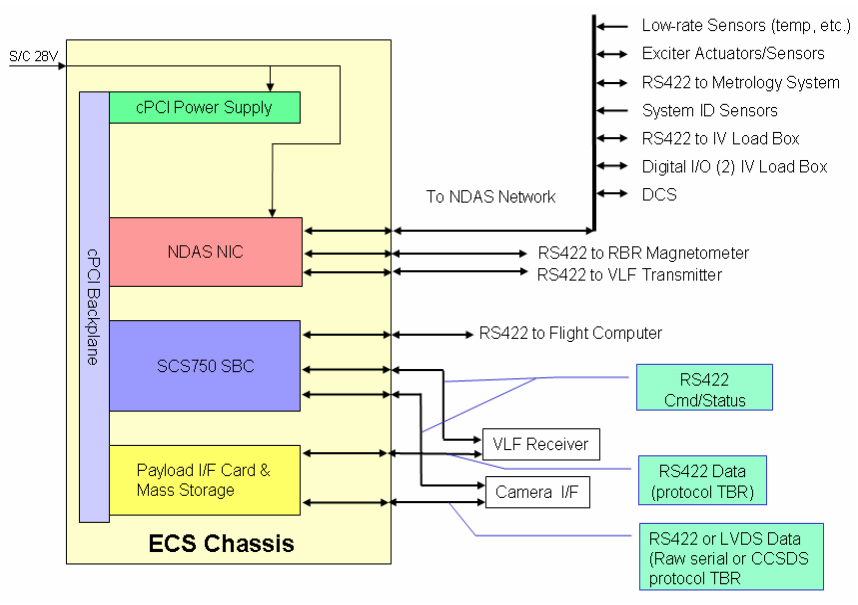

Figure 10 - Experiment Computer System Block Diagram 
The top-level architecture of the existing NDAS architecture (Figure 11) consists of two major elements:

- Network Interface Card (or NIC), which acts as the master of the NDAS bus and provides connectivity of the bus to the flight computer or payload experiment computer system. The figure shows a payload computer labeled ECS.

- Network Node, which interfaces to the network client (e.g., sensor and/or actuator suite) through a variety of analog and digital I/O lines on the one side and connects the NDAS bus on the other. A concept network node envelope drawing is shown in Figure 12.

The NDAS bus is dual redundant for both signal and power. It is based on a low-voltage differential signal (LVDS) physical layer, with a high-definition data link control (HDLC) data link or protocol layer. The data rate is sized for $25 \mathrm{Mbits} / \mathrm{sec}$, based on current throughput and latency requirements of real-time experiments to be conducted on DSX.

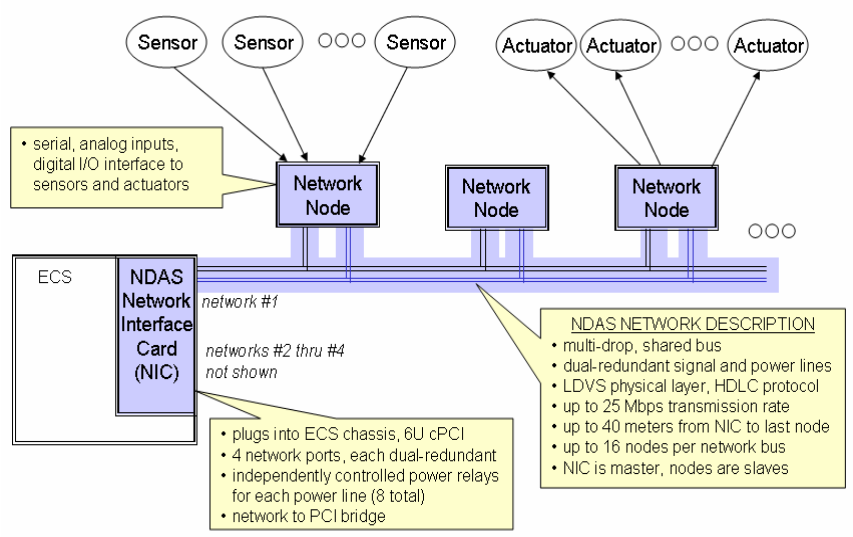

Figure 11 - Network Data Acquisition System Architecture

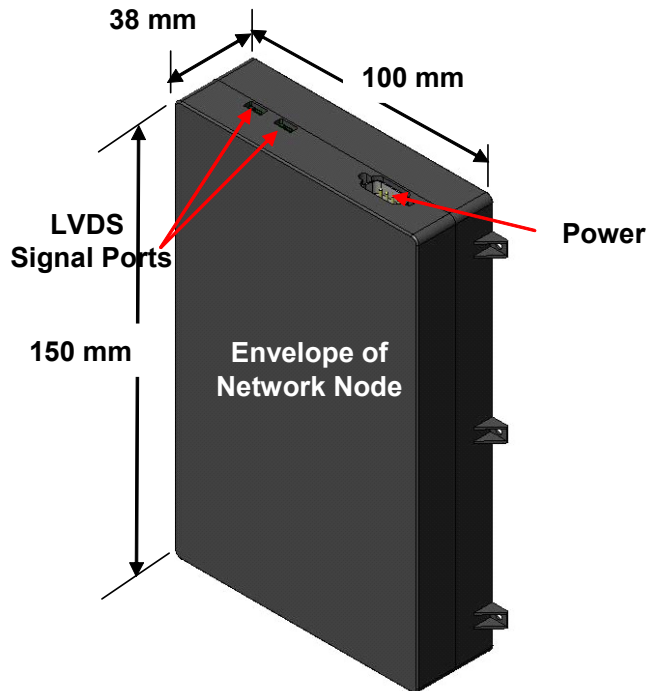

Figure 12 - Network Node Prototype Design Envelope

These modular avionics technologies complement our highly launchable ESPA-based mechanical design; together, they represent a path finder for future rapid-response spacecraft. The next evolutionary step for this technology involves enhancing the network and flight control computer so they are preprogrammed to self-configure spacecraft subsystems, components, devices, and payloads. This step allows for rapid configuration and integration of off-theshelf modular spacecraft consisting of predefined building blocks "on the fly." PSI is funded to evaluate and develop rapid response technologies for space applications under an AFRL Phase I SBIR.

\section{Conclusions}

AFRL has initiated a new flight experiment to perform basic research in the areas of

1. Large deployable structures

2. System identification and adaptive control

3. Radiation belt remediation using VLF waves

4. Thin-film photovoltaics

5. Space weather

DSX is currently under development at AFRL Space Vehicles Directorate, and has recently completed its Systems Requirements Review (SRR). DSX is scheduled to be launch ready in late 2009 .

DSX will provide an important database for future designers of space missions with large, flexible, deployable structures, a high power requirement, and MEO operations. Beyond the inherent value of the scientific experiments, DSX is also path-finding a new, responsive approach for integrating spacecraft. The ESPA platform ensures 
affordable and frequent opportunities for launch. In addition, the combination of a dedicated payload/experiment computer and a network data bus allows a "standard" host spacecraft bus to be used, limiting the development risk to the experiments. The network data bus is the first step leading to the prospect of a "plug-and play" payload and bus modules with script-driven interfaces that are independently verified and developed, and then integrated into highly capable spacecraft.

\section{REFERENCES}

[1] Goodwin, J.S. and Wegner, P., "Evolved Expendable Launch Vehicle Secondary Payload Adapter - Helping Technology get to Space," AIAA Space 2001 Conference and Exposition, AIAA Paper 2001-4701, August 2001.

[2] EELV Standard Interface Group, Evolved Expendable Launch Vehicle Standard Interface Specification Version 6, Kendall, Randy, ed., September 2000.

[3] Heald, J.C. and Peterson, L.D., "Sensitivity of the Deployment Repeatability of a Precision Telescope Mechanism," 43th AIAA/ASME/ASCE/AHS/ASC Structures, Structural Dynamics, and Materials Conference, Denver, CO, April 2002, AIAA Paper 20021502.

[4] Adler, A.L., Hague, N., Spanjers, G., Engberg, B., Goodding, J., Murphy, D.M., and Mikulas, M.M., "PowerSail: The Challenges of Large, Planar, Surface Structures for Space Applications," 44th AIAA/ASME/ASCE/AHS/ASC Structures, Structural Dynamics, and Materials Conference, Norfolk, VA, April 2003, Paper AIAA-2003-1823.

[5] Gussenhoven, M.S., Mullen, E.G., and Brautigam, D.H., "Improved Understanding of the Earth's Radiation Belts from the CRRESS Satellite," IEEE Transactions on Nuclear Science, April 1996, Vol. 43, no. 2, pp.353-368.

[6] Burch, J.L., "IMAGE Mission Overview", Space Science Reviews, January 2000, vol. 91, no. 1-2, pp.1-14.

[7] Martin, M., Klupar, P., Kilberg, S., and Winter, J., "TechSat 21 and Revolutionizing Space Missions Using Microsatellites," USU Small Satellite Conference, 2001.

[8] Winter, J. and Anderson, N., "Distributed Aperture Implementation On the TechSat 21 Satellites," IEEE Aerospace Conference, 2003.
[9] Guidice, D.A., Davis, V.A., Curtis, H.B., Ferguson, D.C., Hastings, D.E., Knight, F.L., Marvin, D.C., Ray, K.P., Severance, P.S., Soldi, J.D., and Van Riet, M., "Photovoltaic Array Space Power Plus Diagnostics (PASP-Plus) Experiment," Final Report, PL-TR-97-1013, Phillips Laboratory Space Technology Directorate, March 1997.

[10] Dichter, B.K., McGarity, J.O. Oberhardt, M.R., Jordanov, V.T., Sperry, D.J., Huber, A.C., Pantazis, J.A., Mullen, E.G., Ginet, G., and Gussenhoven, M.S., "Compact Environment Anomaly Sensor (CEASE): A Novel Spacecraft Instrument for In Situ Measurements of Environmental Conditions," IEEE Trans. Nucl. Sci., 45, 2800, 1998.

[11] Dichter, B.K., Turnbull, W.R., Brautigam, D.H., Ray, K.P., and Redus, R.H., "Initial on-orbit results from the Compact Environmental Anomaly Sensor (CEASE)," IEEE Trans. Nucl. Sci., vol. 48, pp. 2022 - 2028 December 2001. 